\title{
The Influence OF Personality TyPe On TeAmWork IN ENGINEERING EDUCATION
}

\author{
Peter M. Ostafichuk and Carol Naylor \\ University of British Columbia \\ ostafichuk@mech.ubc.ca
}

\begin{abstract}
The influence of personality type on various factors relating to engineering education is examined. Personality type was described according to the MyersBriggs Type Indicator (MBTI). Data from a total of seven cohorts (2007 to 2013) in a second year mechanical engineering design course have been analyzed. Decision making on team tests was examined in terms of the MBTI Introversion / Extraversion domain and peer evaluation scores received were examined across all four MBTI domains. Measured differences between students with a preference for Introversion and those with a preference for Extraversion on the level of influence on team decision making was found. A small but statistically significant correlation has also been noted between peer evaluation scores received and a student's preference on the MBTI Judging / Perceiving domain. This is believed to relate to possible perceptions (or misperceptions) that in delaying action or decision making a person with a preference for Perceiving is lazy or disengaged. Differences in peer evaluation scores were not observed for the other three MBTI domains. The results suggest that even with student awareness (through readings) and interventions (through workshops) possible effects of the difference in personality type persist in engineering student teams. The lack of relationship between peer evaluation scores and the remaining three MBTI domains is a favourable outcome in terms of the objectivity of the peer evaluation tools.
\end{abstract}

Keywords: Myers-Briggs Type Indicator, MBTI, personality type, Team-Based Learning, TBL, team dynamics, design education.

\section{INTRODUCTION}

Personality type theory offers one tool to describe patterns, preferences, and tendencies in human behaviour. Among other things, differences in how individuals interact with one another, take in information, and make decisions can be described using type theory. Team work is an essential element of engineering and is increasing expected in higher education by students, employers, and accrediting bodies [1],[3]. Differences in personality type of the members on a team have the potential to improve team effectiveness by increasing diversity of perspectives and behaviours [2],[8],[14], but these same differences may also may increase tension between team members [4],[13].

The Myers-Briggs Type Indicator (MBTI) is a common tool used for describing personality type and it is based on four domains:

- Introversion / Extraversion: inward directed focus or attention (I) versus outward directed focus or attention (E);

- Sensing / iNtuition: observation of tangible and concrete details, often relying on the five senses (S) versus observation the "big picture," often willing to trust hunches and possibilities $(\mathrm{N})$. (Note: the symbol "N" is used for iNtuition as "I" is already used for Introversion);

- Thinking / Feeling: objective decision making based on logic (T) versus subjective decision making based on values (F); and

- Judging / Perceiving: taking action and making decisions to achieve closure $(\mathrm{J})$ versus delaying decisions to collect more information and keep options open (P).

The current study examines student behaviour in a second year mechanical engineering design course in the context of the above four MBTI domains. In the course, students are introduced to the MBTI through readings, a team-formation survey, and a series of workshops. Design teams engage in various collaborative group activities inside and out of the classroom, including team reading quizzes, tutorial exercises, design projects, presentations, and reports. There are also individual elements in the course including individual reading quizzes, exams, and questionnaires. Peer evaluation is used throughout the course in order to determine how team marks influence and individual's grades.

It is often argued, at least anecdotally, by Team-Based Learning practitioners that team decisions are initially made by those with the "loudest voice" but over time the quieter members begin to speak up and contribute more equally. The first objective of this study was to verify or 
refute this argument using objective course data. Likewise, there is an implicit assumption in using peer evaluation in course grading that the results are objective and valid - that is, evaluations are based on a student's actual contributions to a team as opposed to subjective perceptions, coloured in part by their personality type. The second objective of this study was to examine extensive peer evaluation data to identify whether this assumption is valid.

Drawing from required course elements (tests, projects, assignments, questionnaires, and so on), data for a total of seven cohorts from 2007 to 2013 have been collected and analyzed in relation to the various domains of the MBTI. Aspects of performance considered include the influence of the Introversion / Extraversion preference on team test performance and the influence of all four domains on the peer evaluation scores received by students. This builds in part on previous studies [12] that examined student personality type influences on student perceptions of peer evaluation.

The sections below first outline the course context for the study, with details on course format, team formation, peer evaluation, and team dynamics instruction. The methodology for the study is then presented, outlining the assessment of individual's influence on team decision making and the relationship between peer evaluation scores and MBTI preference. The results and discussion section presents the data collected and their interpretation, and is followed by conclusions that summarize the work highlight implications for teams in design engineering education.

\section{COURSE CONTEXT}

This study was conducted in the Department of Mechanical Engineering at the University of British Columbia (UBC) in a second-year mechanical design course (MECH 223). The course is part of the integrated Mech 2 Program introduced in 2004 [9]. The typical course enrollment is 115-125 students and seven cohorts from 2007 to 2013 were considered. The course is delivered using the Team-Based Learning (TBL) approach [8] (details on the course-specific TBL implementation can be found in [5],[6], and [10]). All students attend a common lecture section (i.e. there are approximately 120 students in the classroom at one time) and they are split into four sections for other activities, such as tutorials, team meetings with a teaching assistant, computer labs, and so on. The MECH 223 course is atypical in several respects: first, it is a full-time course that runs for seven weeks (students do not take other courses at the same time); second, the course is split into two parts (four weeks in January and three weeks in April, each with a separate major project); and, third, the course is large in scope at seven credits (a typical course at UBC is three credits).
Following recommended practice, teams of six to seven students (20 teams in the course) were instructorformed [2] in order to maximize diversity [4],[14] and to minimize previously established subgroups [8]. Prior to the course, students completed an abbreviated version of the MBTI online through the TypeFocus tool (http://www.typefocus.com). A mandatory course intake questionnaire then collected each student's MBTI preferences as well as self-reported ability with hand skills, software skills, communication skills, and team skills. The above information was combined with GPA from previous courses to form teams that were heterogeneous across all personality, skill, and GPA criteria. Each team possessed at least two members with each MBTI preference with the exception of the Feeling preference; in most years there were not enough students reporting a preference for Feeling to uniformly distribute them across all teams in light of other team formation constraints. This use of Myers-Briggs types in forming student teams is also used by others; Lester et al. [7] suggest the use of Myers-Briggs as one approach to minimize "disaster groups" in engineering projects. The same project teams were maintained for the course duration, including the January and April sessions.

As part of course requirements, students completed six mandatory peer evaluations using the iPeer online software tool (http://ipeer.ctlt.ubc.ca). Three evaluations were completed using a point-based evaluation in which each student distributed an average of 100 points per other team member (students could reward above average performance with an score above 100, but that required lowering the evaluation scores, on average, for other team members). The other three evaluations were completed using a rubric-based approach in which students evaluated their teammates on four criteria (communication, initiative, responsibility, and professional behaviour) across four levels of mastery (below expectations, marginal, meets expectations, and exceeds expectations). The raw numerical scores for the rubric-based evaluation were normalized on a evaluator-by-evaluator basis to an average of 100 to match the point-based evaluations. Both forms of evaluation required the students to provide comments to justify the scores they assigned. A comparison of each of the two methods was previously reported in [12]. Half of the teams used the point-based evaluation for the first project and rubric-based for the second, and the other half of the teams used the same evaluations in reverse order. After each evaluation, students received aggregate scores and anonymous, randomly ordered comments from their teammates. The average peer evaluation score for each student at the end of the term was multiplied against their team's net grade in order to determine the individual portion of the team grade recorded for that student.

Lastly, as part of the design course, students were introduced to the MBTI and team dynamics in multiple 
ways. Students completed a short textbook reading (4 pages) on the MBTI and its implications for team work at the start of the course. At the start of each project they also participated in a team dynamics workshop (one in January and one in April). The first workshop focused on understanding the MBTI and developing strategies for working as a team based on the distribution of types on each team. The second workshop focused on debriefing the experiences from the first project and then developing strategies for giving and receiving feedback and managing conflict. Further information on the team dynamics workshops is available in reference [11].

\section{METHODOLOGY}

The influence of personality type on team work was considered by attempting to identify differences in test performance or peer evaluation scores based on students' MBTI preferences. The specific elements that were considered include:

- Chance of being influenced: the likelihood that an individual would arrive at the correct answer to a question on a reading quiz but the team response to the same question would be a different (incorrect) answer was measured. This was examined in the context of the Introversion / Extraversion MBTI domain; type theory suggests a student with a preference for Introversion would be less outspoken and more likely to fall into this category.

- Chance of influencing others: the likelihood that an individual would arrive at an incorrect answer to a question on a reading quiz and the team response to the same question would be the same incorrect answer was measured. This also was examined in the context of the Introversion / Extraversion MBTI domain; type theory suggests a student with a preference for Extraversion would be more outspoken and more likely to fall into this category.

- Peer evaluation scores received: the average peer evaluation scores assigned by teammates was compared for each of the four MBTI domains. Several differences that could potentially boost evaluation scores on average could be anticipated based on type theory: those with a preference for Extraversion could be viewed as "more social;" those with a preference for Sensing could be viewed as more practical or focused on project details; those with a preference for Feeling could be seen as warmer or more caring; and those with a preference for Judging could be seen as possessing greater drive or follow-through.

The methodology to examine the above three elements is outlined further below.

\subsection{Measuring Influence by or on Others}

As part of the TBL teaching approach, students complete a set of assigned readings for each course module and then take an in-class quiz on those readings. The reading quiz is completed prior to any classes, tutorials, or other teaching activities on the reading topics. The reading quiz consists of usually 20 questions, all multiple choice. Of particular importance in the current context, students first complete the reading quiz individually by marking their responses on a computer score card, and then they complete the quiz a second time in their teams. The team quiz questions are identical to those on the individual test, but this time responses are recorded using an Immediate Feedback Assessment Technique (IF-AT) scratch card, as shown in Fig. 1.

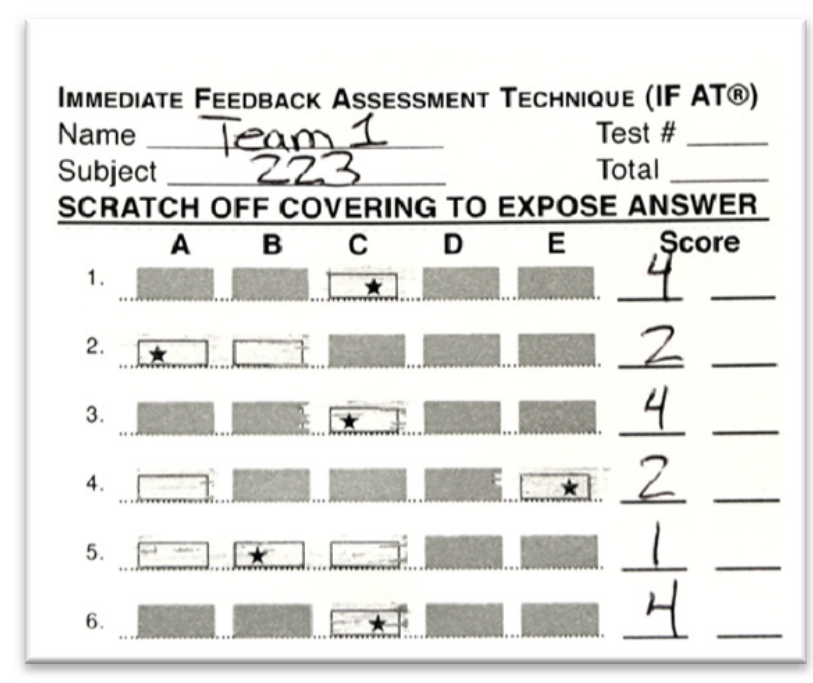

Fig. 1. IF-AT card for the team reading quiz

On the IF-AT card questions are numbered as rows down the left side and answer choices are labelled as columns across the top of the card. Teams discuss the question and scratch the box corresponding to their response. If they are correct they reveal a star symbol $(\star)$, such as for Question 1, C, in the figure. If teams are incorrect, no star is revealed (such as Question 2, B, in the figure), and then teams discuss the question further and try again until they reveal the correct answer (Question 2, A). The score per question is based on the number of scratches to reveal the star. (In MECH 223: 1 try $=4$ points; 2 tries $=2$ points; 3 tries $=1$ point; and 4 or 5 tries $=0$ points.)

Having both individual and team responses to the exact same questions on tests taken immediately after one another allows comparison of how those with preferences for Introversion and Extraversion behave. Specifically, 
the IF-AT card reveals cases where the team is incorrect (such as Question 2, answer B, in Fig. 1) and the computer-scored individual tests, taken prior to the team test, reveal how each person responded to the same question without the influence of the other team members.

In total, over 8,800 question events have been analyzed from the 2008 to 2011 offerings of the course. This is based on 24 quizzes, 10-20 questions per quiz, and 20 teams per year. A total of 896 incorrect team responses to questions were identified.

\subsection{Peer Evaluation Scores Received}

The peer evaluation scores received were examined based on the evaluatees' MBTI preferences. A separate statistical comparison based on a two-tailed t-test for the difference in means was completed for each of the four MBTI domains. Each cohort was considered separately first and then aggregate results were examined for all cohorts together. The sample sizes by cohort and the distribution of types within each cohort as summarized in Table 1 below. As described in Section 2, each student completed a total of six peer evaluations in which they evaluated each other member on their team.

Table 1: Distribution of MBTI types

\begin{tabular}{cccccccccc}
\hline Cohort & $\mathrm{n}$ & $\mathrm{I}$ & $\mathrm{E}$ & $\mathrm{S}$ & $\mathrm{N}$ & $\mathrm{T}$ & $\mathrm{F}$ & $\mathrm{J}$ & $\mathrm{P}$ \\
\hline 2012W & 124 & 57 & 67 & 50 & 74 & 88 & 36 & 68 & 56 \\
2011W & 128 & 67 & 61 & 53 & 75 & 98 & 30 & 66 & 62 \\
2010W & 114 & 64 & 50 & 55 & 59 & 95 & 19 & 66 & 48 \\
2009W & 118 & 62 & 56 & 55 & 63 & 98 & 20 & 78 & 40 \\
2008W & 111 & 55 & 56 & 42 & 69 & 91 & 20 & 61 & 50 \\
2007W & 106 & 54 & 52 & 44 & 62 & 82 & 24 & 63 & 43 \\
2006W & 112 & 67 & 45 & 51 & 61 & 92 & 20 & 66 & 46 \\
All & 813 & 427 & 386 & 348 & 465 & 644 & 169 & 471 & 342 \\
\hline
\end{tabular}

\section{RESULTS AND DISCUSSION}

The sections below present the results and discussion for the points raised at the start of Section 3: what is the likelihood a student will be influenced by the team during team decision making, what is the likelihood a student will influence others during team decision making, and do peer evaluation scores received by a student have any relationship to personality preference as described by the MBTI.

\subsection{Likelihood of Being Influenced by Others}

Based on the individual and team reading quizzes described in Section 3.1 above, the likelihood that an individual with a correct answer was influenced to go along with the team in choosing an incorrect response was quantified by the ratio below,

$$
\frac{\text { Instances I-types change answer } / \mathrm{n}_{I}}{\text { Instances E-types change answer } / \mathrm{n}_{E}}
$$

where $\mathrm{n}_{I}$ is the total number of students expressing a preference for Introversion and $\mathrm{n}_{E}$ is number expressing a preference for Extraversion. No difference between the two groups on average corresponds to a ratio of unity.

The results in Fig. 2 below show, for the cohorts studied, that students with a preference for Introversion are on average $16 \%$ more likely than their Extraversion counterparts to be influenced to go along with the team when they have the correct answer. This phenomenon is present for all four years for which data has been analyzed, and discrepancy ranges from a low of $6 \%$ to a high of $37 \%$. This is in spite of the interventions and strategizing through team dynamics workshops to highlight this potential issue. Perhaps more concerning, this issue is more prevalent in the second half of the course than the first (the overall averages change from 1.10 for January to 1.23 for April).

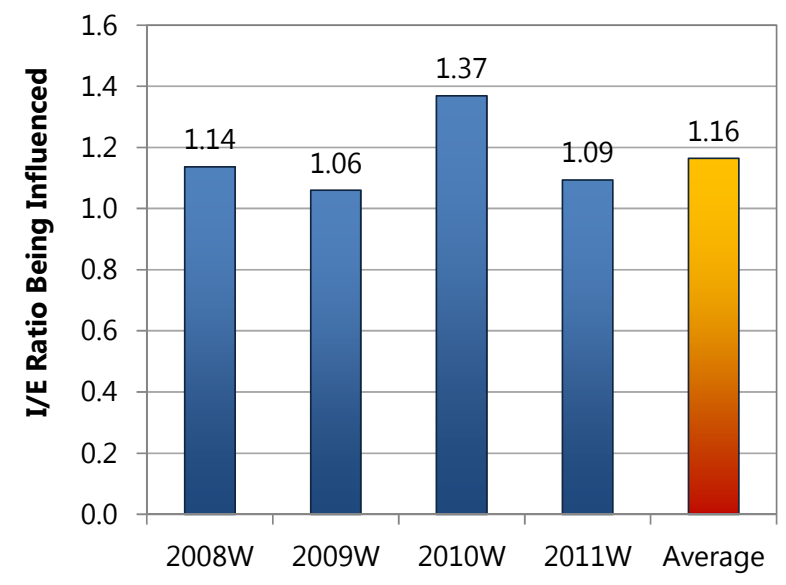

Fig. 2. Ratio of I-types influenced to E-types influenced

These results suggest that if teams worked harder to engage members with a preference for Introversion (that is, less outspoken individuals), the team would benefit but up to $16 \%$ fewer incorrect decisions on average. The results also suggest that the commonly held belief among TBL practitioners that Introversion / Extraversion differences in decision making diminish over time as the team develops is false.

\subsection{Likelihood of Influencing Others}

Considering the opposite case where a student has an incorrect answer and the team selects the same incorrect answer, the assumption is made that the individual played 
Proc. 2013 Canadian Engineering Education Association (CEEA13) Conf.

a role the influencing the team decision. In this case the effect is quantified by the ratio below where $\mathrm{n}_{E}$ and $\mathrm{n}_{I}$ are as defined above. Again, no difference between the groups corresponds to a ratio of unity.

$\frac{\text { Instances E-types and team have same wrong answer } / \mathrm{n}_{E}}{\text { Instances I-types and team have same wrong answer } / \mathrm{n}_{I}}$

The results in Fig. 3 show that in instances where the team selects an incorrect answer it is consistently more likely that a student with a preference for Extraversion also had that same incorrect answer. For the cohorts studied, this effect ranges from almost no difference $(1 \%)$ to a high of $20 \%$ and an average of $8 \%$.

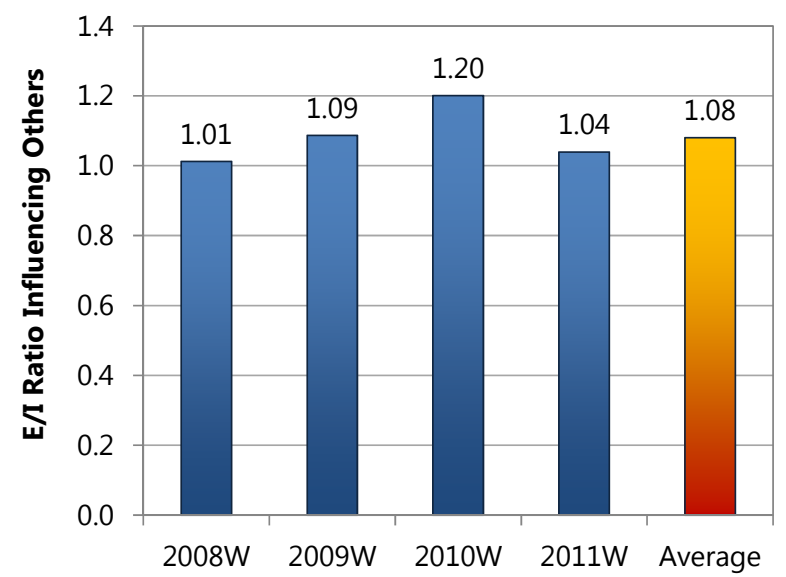

Fig. 3. Ratio of E-types influencing others to l-types influencing others

These results are consistent with those from Section 4.1 and they suggest that if teams made more of an effort to balance input from more outspoken members (those with a preference for Extraversion) with input from less outspoken members (preference for Introversion), the team would benefit with fewer incorrect decisions. Also consistent with the previous findings, this effect became more significant as the course progressed (the ratio grew from 1.05 in January to 1.12 in April).

\subsection{Differences in Peer Evaluation Scores}

Average peer evaluation scores for each MBTI domain are shown below in Table 2 through Table 5 along with the $\mathrm{p}$-value corresponding to a two-tailed t-test for the difference in means. Instances where the measured difference can be considered statistically significant $(\mathrm{p} \leq$ 0.05 ) are identified with the p-value bolded and italicized.
Table 2: Peer evaluation scores for Introversion / Extraversion

\begin{tabular}{cccc}
\hline Cohort & I & E & p \\
\hline $2012 W$ & 100.08 & 99.92 & 0.638 \\
$2011 W$ & 100.21 & 99.79 & 0.340 \\
$2010 W$ & 100.08 & 99.92 & 0.731 \\
$2009 W$ & 100.07 & 99.93 & 0.320 \\
$2008 W$ & 100.37 & 99.63 & 0.173 \\
$2007 W$ & 99.98 & 100.02 & 0.901 \\
$2006 W$ & 99.65 & 100.35 & 0.146 \\
Combined & 100.09 & 99.91 & 0.228 \\
\hline
\end{tabular}

Table 3: Peer evaluation scores for Sensing / iNtuition

\begin{tabular}{cccc}
\hline Cohort & $\mathbf{S}$ & $\mathbf{N}$ & $\mathbf{p}$ \\
\hline 2012W & 99.81 & 100.19 & 0.285 \\
2011W & 100.50 & 99.50 & $\mathbf{0 . 0 2 1}$ \\
2010W & 100.40 & 99.60 & 0.085 \\
2009W & 100.10 & 99.90 & 0.131 \\
2008W & 99.81 & 100.19 & 0.496 \\
2007W & 100.17 & 99.83 & 0.226 \\
2006W & 100.01 & 99.99 & 0.975 \\
Combined & 100.14 & 99.86 & 0.060 \\
\hline
\end{tabular}

Table 4: Peer evaluation scores for Thinking / Feeling

\begin{tabular}{cccc}
\hline Cohort & $\mathbf{T}$ & $\mathbf{F}$ & $\mathbf{p}$ \\
\hline 2012W & 100.34 & 99.66 & $\mathbf{0 . 0 5 0}$ \\
2011W & 99.70 & 100.30 & 0.166 \\
2010W & 99.70 & 100.30 & 0.188 \\
2009W & 100.11 & 99.89 & 0.102 \\
2008W & 100.13 & 99.87 & 0.625 \\
2007W & 100.18 & 99.82 & 0.205 \\
2006W & 100.02 & 99.98 & 0.945 \\
Combined & 100.06 & 99.94 & 0.421 \\
\hline
\end{tabular}

Table 5: Peer evaluation scores for Judging / Perceiving

\begin{tabular}{cccc}
\hline Cohort & J & P & p \\
\hline 2012W & 100.45 & 99.55 & $\mathbf{0 . 0 1 0}$ \\
2011W & 100.86 & 99.14 & $<\mathbf{0 . 0 0 1}$ \\
2010W & 100.71 & 99.29 & $\mathbf{0 . 0 0 2}$ \\
2009W & 100.12 & 99.88 & $\mathbf{0 . 0 7 3}$ \\
2008W & 100.81 & 99.19 & $\mathbf{0 . 0 0 3}$ \\
2007W & 100.02 & 99.98 & 0.897 \\
2006W & 100.25 & 99.75 & 0.310 \\
Combined & 100.49 & 99.51 & $<\mathbf{0 . 0 0 1}$ \\
\hline
\end{tabular}


As shown, no statistically significant differences were observed for the first three MBTI domains (Introversion / Extraversion, Sensing / iNtuition, and Thinking / Feeling). In the Judging / Perceiving domain, statistically significant differences were observed in five of the seven cohorts as well as all cohorts combined. Also of note, the observed differences of students with a preference for Judging receiving higher peer evaluation scores on average than those with a preference for Perceiving are true for all seven of the cohorts studied. As shown, the average difference in peer evaluation grades is $1.0 \%$.

To rule out other factors besides Judging / Perceiving as contributing to the observed differences in peer evaluation scores, exam performance and lateness in submitting assignments were compared for the two groups. There was no statistically significant difference between the two groups in terms of the likelihood of missing an assignment deadline nor was there a difference on the first of two final exams (testing cognitive ability in comprehension and application in design). Interestingly, there was a statistically significant difference on the second final exam that tested high-level cognitive ability in design (synthesis, evaluation, and judgement) with those with a preference for Perceiving outscoring those with a preference for Judging by nearly $2 \%$. This stronger exam performance is the opposite of what might be expected to explain differences in peer evaluation scores.

The most likely explanation for the difference in peer evaluation scores for the Judging and Perceiving types lies in the perception of task completion by peers. Given those with a preference for Judging are more likely to commit to a decision or course of action, get the job done, and move on, this could be viewed favourably by others. In contrast, those with a preference for Perceiving may delay making a decision or taking action - perhaps for very good reason such as to further think things through, make sure a decision is the right one, or avoid jumping to a conclusion - which ultimately could mistakenly be interpreted as procrastination or laziness. The lack of a difference in submitting required course materials on time and the slightly improved exam scores noted above suggest the students with the preference for Perceiving are at least as capable as the students with the preference for Judging; in other words, peer evaluation score differences appear to be explainable in terms of perception rather than actual ability or contribution.

\section{CONCLUSIONS}

The influence of personality type on several aspects of teamwork in engineering education has been examined. Personality type was classified according to the MBTI and two main impacts were considered: does preference for Introversion or Extraversion affect an individual's influence on team decision making and is there a relationship between an individual's personality preferences (across all four MBTI domains) and the peer evaluation scores that person receives.

In all cases differences were observed. Students with a preference for Introversion were 16\% more likely than their Extraversion counterparts to go along with the team in selecting an incorrect answer to a test after themselves previously correctly answering that same question on an individual test. Similarly, those with a preference for Extraversion were $8 \%$ more likely to have selected the same incorrect answer on an individual test that the team later selected on a team test. Following type theory, it is inferred that those with a preference for Extraversion have a greater influence on the team by virtue of their more outspoken nature. These trends were found to become more pronounced as the course progressed, refuting the claim by some that Introversion / Extraversion differences diminish over time in persistent teams. Importantly, these differences are observed in a course where teams are explicitly made aware of the potential for this effect through workshops and other interventions requiring discussion and strategizing of how to avoid these issues.

Lastly, a statistically significant difference amounting to $1.0 \%$ in peer evaluation scores was observed for those with a preference for Judging compared to those with a preference for Perceiving. Following type theory, it is presumed this difference is due to a perception that those with a preference for Judging are more likely to take action and make decisions more quickly than those with a preference for Perceiving; delaying action or decision making, even with good cause, could mistakenly be interpreted as laziness or procrastination. There were no statistically significant differences observed for the other three domains (Introversion / Extraversion, Sensing / iNtuition, and Thinking / Feeling) at least lending some support to the use of peer evaluation as an objective measure.

\section{References}

[1] ABET, Criteria for Accrediting Engineering Programs, Baltimore, MD: Accreditation Board for Engineering and Technology (ABET), 2012, 26 pp. Available as of May 3, 2013 from: http://www.abet.org/uploadedFiles/Accreditation/ Accreditation_Step_by_Step/Accreditation_Documents/Current/ 2013_-_2014/eac-criteria-2013-2014.pdf

[2] James Brickell, Avid Porter, Michael Reynolds, and Richard Cosgrove, "Assigning Students to Groups for Engineering Design Projects: A Comparison of Five Methods," Journal of Engineering Education, Vol. 83, Issue 3, pp. 259-62, July 1994.

[3] CEAB, Accreditation Criteria and Procedures: Canadian Engineering Accreditation Board (CEAB), 2012, 114 pp. Available as of May 3, 2013 from: http://www. 
Proc. 2013 Canadian Engineering Education Association (CEEA13) Conf.

engineerscanada.ca/files/w_Accreditation_Criteria_Procedures 2012.pdf

[4] Susan Feichtner and Elaine Davis, "Why Some Groups Fail: A Survey of Students' Experiences with Learning Groups," The Organizational Behaviour Teaching Review, 9 (4), pp. 58-73, 1984.

[5] Anthony Hodgson and Peter Ostafichuk, "TeamBased Learning in the Design Modules of a New, Integrated, 2nd Year Curriculum at UBC," in Proc. CDEN 2005 (Kananaskis, AB, 17-20 July, 2005), 9 pp., 2005.

[6] Antony Hodgson and Peter Ostafichuk, "Designing Extended Assignments for Team-Based Learning Modules," in Proc. CDEN 2006 (Toronto, ON, 24-26 July 2006), 10 pp., 2006.

[7] Edward Lester, Damian Schofield, and Peter Chapman, "The interaction of engineering 'types': A study of group dynamics and its relationship to self and peer assessment during computer-based exercises", Engineering Education, Vol. 1, No. 1, pp. 39-49, 2006.

[8] Larry Michaelsen, Michael Sweet, and Dean Parmelee, Team-Based Learning: Small Group Learning's Next Big Step. New Directions for Teaching and Learning, JosseyBass, San Francisco, 129 pp. \{ISBN: 978-0470462126\}

[9] Peter Ostafichuk, Elizabeth Croft, Sheldon Green, Gary Schajer, and Steven Rogak, "Analysis of Mech 2: An
Award-Winning Second Year Mechanical Engineering Curriculum," in Proc. of EE2008 (Loughborough, UK, 14-16 July, 2008), 12 pp., 2008.

[10] Peter Ostafichuk and Anthony Hodgson, "Standing on Our Heads: How Teaching Engineering Design Looks Different from a Team-Based Learning Perspective", in Proc. TeamBased Learning Conference 2007 (Vancouver, BC, 31 May - 1 June, 2007), 36 pp., 2007.

[11] Peter Ostafichuk, Anthony Hodgson, Sophie Bartek, and Carol Naylor, "Teaching Team Dynamics: Experiences in Second Year Mechanical Engineering Design", in Proc. CDIO Conference (Montreal, QC, 14-17 June, 2010), 10 pp., 2010.

[12] Peter Ostafichuk and Claire Jones, "Assessing Peer Evaluation: A Comparative Study of Two Peer Evaluation Methods Used in an Engineering Design Course", in Proc. 6th International Conference on Innovation and Practices in Engineering Design and Engineering Education, (Hamilton, ON, 2009), 6 pp., 2009.

[13] Maryellen Weimer, "Why Groups Fail: Student Answers," The Teaching Professor, Vol. 5, No. 9, November 1991.Wright, D., 1994, Using Learning Groups in Your Classroom: A Few How-To's, Teaching at UNL (University of Nebraska-Lincoln), 15 (4).

CEEA13; Paper 035

Montreal, QC; June 17-20, $2013 \quad-7$ of 7 - 